\title{
Diskussion
}

\author{
Vilmos Ágel
}

\section{Analyse und Synthese - ein Diskussionsbeitrag}

\author{
Abstract \\ The historical linguistic topos stating that German has developed from a synthetic language \\ to an analytic or more analytic language has been not only critically questioned in recent \\ work by Damaris Nübling and Renata Szczepaniak, but even reversed into its opposite. It \\ is claimed that German has become not only paradigmatically but also syntagmatically \\ more synthetic. A point of particular interest in this view is that it is not based on new, \\ hitherto unknown historical linguistic facts, but rather reinterprets the known facts. \\ In the present contribution this reinterpretation will be put into question and the no- \\ tional and terminological possibilities will be searched for that do justice to both the syn- \\ thetic and the analytic tendencies, while leaving a relatively broad space for theory-specific \\ interpretations. The conclusion of the discussion is a modified representation of the most \\ important morphological language types. \\ 1. Diskussionsanlass \\ 2. Das Nübling/Szczepaniak-Modell \\ 3. Diskussion \\ 3.1 Unterschiedliche Begriffe von Analyse/Synthese \\ 3.2 Der Begriff des kombinierenden (diskontinuierenden) morphologischen Sprach- \\ typs \\ 3.2.1 Das Verfahren der syntagmatischen Disambiguierung \\ 3.2.2 Periphrasen im Sinne des NSM \\ 3.2.3 Kombinatorik und Klammerung \\ 3.2.4 Zwischenfazit: Was bleibt von der syntagmatischen Synthetisierung übrig? \\ 4. Morphologische Sprachtypen und die Modellierung des Sprachwandels \\ 5. Literatur
}

\section{Diskussionsanlass}

Die Diskussion um den in elaborierter Form auf Edward Sapir (1921: 128) zurückgehenden „degree of synthesis“ (ebd.: viii) hat mit neueren Arbeiten von Damaris Nübling und Renata Szczepaniak (Nübling 2010; Szczepaniak 2010) eine überraschende Wende genommen: Der „sprachhistorische Topos“, dass sich das Deutsche von einer synthetischen zu einer analytischen oder analytischeren Sprache entwickelt habe (Nübling 2010: 1), wird nicht nur - zu Recht - kritisch hinterfragt, sondern - m. E. zu Unrecht - in sein Gegenteil gekehrt. Besonders span- 
nend an dieser Auffassung, die ich im Folgenden das Nübling/SzczepaniakModell (kurz: NSM) nennen werde, ist, dass sie nicht auf neuen, bisher unentdeckten sprachhistorischen Fakten basiert, sondern dass sie die bekannten Fakten in ein anderes Licht rückt. Diese Neuinterpretation enthält einerseits eine Reihe von anregenden und weiterführenden Überlegungen wie z. B. die Berücksichtigung der paradigmatischen Achse und der Tokenfrequenz bei der Beurteilung des Synthesegrades. Andererseits stellt sich die Frage, ob man nicht das Kind mit dem Bade ausschüttet, wenn man nicht nur die unbestritten vorhandenen paradigmatischen und frequenziellen Synthetisierungstendenzen betont, sondern auch die syntagmatischen Analytisierungstendenzen als Synthetisierungstendenzen reinterpretiert.

Im vorliegenden Beitrag wird ausgehend vom NSM (Abschnitt 2) nach begrifflichen und terminologischen Möglichkeiten gesucht, die sowohl den Synthetisierungs- als auch den Analytisierungstendenzen gerecht werden und die theoriespezifischen Interpretationen (relativ) weiten Spielraum lassen (Abschnitt 3). Das Fazit der Diskussion ist eine modifizierte Darstellung der wichtigsten morphologischen Sprachtypen (Abschnitt 4).

\section{Das Nübling/Szczepaniak-Modell}

Die kurze Rekapitulation des NSM soll mit der Übersicht der nach Nübling vier wichtigsten morphologischen Sprachtypen begonnen werden (Abbildung 5 in Nübling 2010: 9):

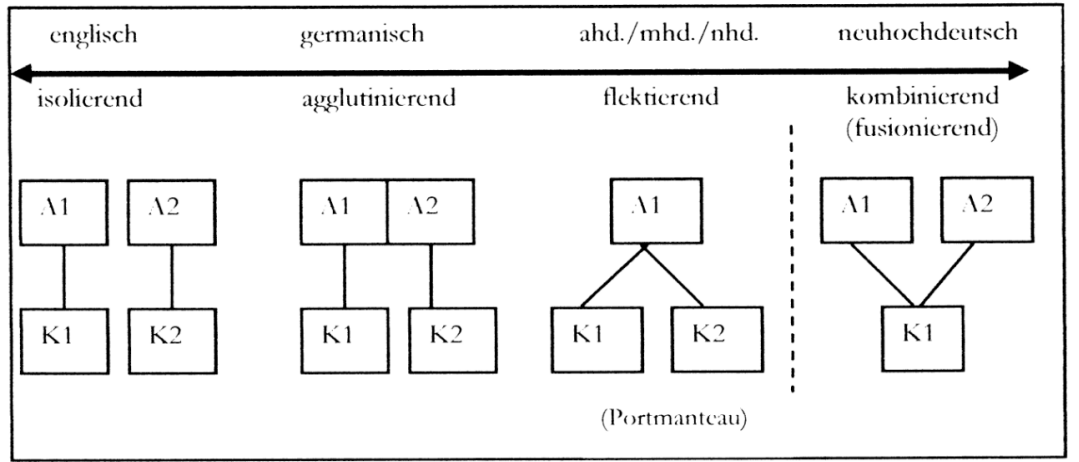

Abb. 1: morphologische Sprachtypen nach Nübling 2010

Die Typologie basiert auf der Zuordnung von Ausdruck $(=\mathrm{A})$ und Inhalt $(\mathrm{K}=$ Kategorie). Die einzelnen Sprachtypen sind von links nach rechts nach zunehmendem Synthesegrad angeordnet: Am analytischsten ist demnach der isolierende Typ, dem u. a. Englisch angehört, am synthetischsten der kombinierende Typ, dem Neuhochdeutsch zugerechnet wird. Nach dieser Skala sind also Deutsch und 
Englisch typologisch maximal unterschiedlich. Der kombinierende Typ wird in Klammern auch fusionierend genannt (worauf in Abschnitt 3 noch zurückzukommen sein wird). Der kombinierende (oder: diskontinuierende) Typ wird in Anlehnung an Arbeiten von Otmar Werner und Elke Ronneberger-Sibold (s. etwa Werner 1979 und Ronneberger-Sibold 1980) als ein innerhalb einer bestimmten syntaktischen Einheit (die nicht nur Phrase sein kann) interdependentes morphologisches Kodierungsverfahren begriffen, bei dem es keine kongruierenden Affixe gibt, die lediglich redundante grammatische Informationen wiederholen (s. span. la-s casa-s roja-s (Nübling 2010: 9)), sondern die Affixe „eröffnen ambige Optionen, die erst durch weitere Affixe vereindeutigt werden“ (ebd.) Das Hauptmerkmal des kombinierenden Typs ist also, dass Kongruenz zu Diskontinuität geworden ist.

Szczepaniak, die das NSM an der Geschichte der deutschen Nominalgruppe exemplifiziert, spricht hier von syntagmatischer Verdichtung: „Die folgende Analyse der NP-Entwicklung vom Althochdeutschen (Ahd.) zum Neuhochdeutschen (Nhd.) wird zeigen, dass man zu keinem Zeitpunkt von einer eindeutigen Analytisierung sprechen kann. Vielmehr wird der Grad an syntagmatischer Verdichtung kontinuierlich erhöht, dadurch dass morphosyntaktische Informationen zunehmend diskontinuierlich, also in Kooperation von ambigen Flexionsendungen ausgedrückt werden, z. B. G.Pl. in d-er (ambig) schön-en (ambig) Katze-n (ambig).“ (Szczepaniak 2010: 124)

Interpretiert wird die syntagmatische Verdichtung als hoher Synthesegrad: „Der hohe Synthesegrad der neuhochdeutschen NP ergibt sich aus der diskontinuierlichen (oder kooperativen) Flexion ihrer Elemente. Der Wandel von einer kongruierenden NP im Althochdeutschen zu einer diskontinuierlichen im Neuhochdeutschen hat dazu geführt, dass sich heute kaum noch Kongruenzfälle finden lassen. Nur noch im Singular tritt die eindeutige G.Sg.Nicht-Fem.-Form des neben der eindeutigen Substantivform Teppich-s auf.“ (Szczepaniak 2010: 132)

Was die Proportion von Kongruenz- und Diskontinuitätsfällen im Neuhochdeutschen anbelangt, scheinen sich Nübling und Szczepaniak jedoch nicht ganz einig zu sein. Nach Nübling (2010: 10) liegt „,der entscheidende Unterschied der Diskontinuität zur Kongruenz in der Ambiguität beider Elemente." Diskontinuierliche Kategorienrealisierung liegt folglich bei sie komm-t und sie komm-en vor, kongruierende dagegen bei du komm-st, er komm-t und wir komm-en (ebd.) Hinzugefügt werden könnten die ebenfalls kongruierenden Formen ich komm-e, es komm-t und ibr komm-t, was bedeutet, dass nach Nübling in diesem verbalen Segment zwei diskontinuierenden sechs kongruierende Formen gegenüberstehen.

Im Gegensatz zu Nübling spricht Szczepaniak (2010: 132) nur dann von Kongruenz, wenn beide Formen eindeutig sind. Zu Diskontinuität reicht ein ambiges Element (= schwache Diskontinuität), zwei ambige Elemente begründen eine noch intensivere Diskontinuität (= starke Diskontinuität). Nach ihr wären also nur ich komm-e und du komm-st kongruierend, alle anderen Formen schwach oder 
stark diskontinuierend. Dies bedeutet, dass nach Szeczepaniak in diesem verbalen Segment zwei kongruierenden sechs diskontinuierende Formen gegenüberstehen.

Die Suche nach Interdependenzen bleibt nicht bei den syntagmatischen Relationen (Affixrelationen bzw. Relation von Subjektspronomen und Verbflexiv) stehen. In Anlehnung an Armin Schwegler (1990) werden auch Periphrasen, die ausdrücklich von analytischen Verbformen unterschieden werden, in das kombinierende Verfahren mit einbezogen. So ergibt sich etwa die Kategorie ,Passiv' erst aus der Kombination von Hilfsverb und Partizip II (Nübling 2010: 11). Durch die Subsumierung der Periphrase als Untertyp des kombinierenden Verfahrens erhalten Periphrasen, d. h. die Konstruktionen, die den „sprachhistorischen Topos“, dass sich das Deutsche von einer synthetischen zu einer analytischen oder analytischeren Sprache entwickelt habe, begründet haben, automatisch den Status von hochgradig synthetischen Konstruktionen.

Das Fazit in Bezug auf die syntagmatische Achse lautet: „Die zentrale Frage [...] ist, ob das diskontinuierende (kombinierende) Verfahren als synthetischer zu gelten hat als das flektierende. [Diese Frage] ist [...] eindeutig zu bejahen: Durch das kombinierende Verfahren nimmt die syntagmatische Binnenverdichtung, die morphologische Interdependenz noch ungleich stärker zu als bei der bloßen Kongruenz als einem Wiederholungsphänomen; beim kombinierenden Verfahren werden nicht einfach nur bereits gesetzte Informationen repliziert, sondern überhaupt erst konstituiert. “ (Nübling 2010: 12)

Nimmt man die auf der paradigmatischen Achse zu Recht festgestellten Synthetisierungstendenzen hinzu, ergibt sich ein (nicht nur im Bereich der Nominalgruppe) relativ eindeutiges sprachtypologisches Bild: „Misst man den Grad der Synthese auf paradigmatischer und syntagmatischer Ebene, so ist festzustellen, dass in beiden Dimensionen die Synthetizität zunimmt. Auf der paradigmatischen Achse ist 1) der Kategorienzuwachs (Definitheit) und 2) die vermehrte Pluralallomorphie zu verzeichnen. Der Formzusammenfall ist hingegen maßgeblich an der syntagmatischen Verdichtung beteiligt. Er führt zum Wandel von der Kongruenz zur Diskontinuität, die die Struktur der neuhochdeutschen NP bestimmt (Werner 1979). Da keine grammatische Kategorie abgebaut wird, müssen ambige Formen miteinander kooperieren. Versteht man also Synthese als Form der Informationsbündelung, so muss man folgern, dass die deutsche NP als Ganzes hochgradig synthetisch organisiert ist.“ (Szczepaniak 2010: 133f.)

\section{Diskussion}

\subsection{Unterschiedliche Begriffe von Analyse/Synthese}

Interpretiert man die herkömmlich als Analytisierung aufgefassten Tendenzen als Synthetisierungstendenzen, stellt sich als erstes die Frage, ob im Rahmen der Neuinterpretation mit denselben Begriffen von Analyse und Synthese gearbeitet 
wird wie im Rahmen der traditionellen Auffassung, die im Folgenden als TA abgekürzt werden soll.

Im NSM wird mit dem primär romanistisch fundierten Konzept von Armin Schwegler gearbeitet, nach dem Analytizität semantische, syntaktische, morphologische und phonologische ,autonomy of morphemes within a speech unit“ und Synthetizität semantische, syntaktische, morphologische und phonologische „interdependency (or relatedness) of morphemes within a speech unit“ darstellt (Schwegler 1990: XV). Verortet man im Rahmen dieses Konzepts, das Synthese als morphologische Interdependenz begreift, Werners und Ronneberger-Sibolds diskontinuierenden/kombinierenden Sprachtyp, kommt man notwendigerweise zu dem Schluss, dass der kombinierende Typ hochgradig synthetisch ist.

Schweglers Konzept stellt allerdings nicht den theoretischen Rahmen dar, in dem sich die von Nübling und Szczepaniak kritisierte TA bewegt. Deren Grundlage ist nicht die Autonomie/Interdependenz von Morphemen, sondern deren Freiheit/Gebundenheit: „Von einer synthetischen Form spricht man, wenn verschiedene Elemente zu einer Form verschmelzen. Dies ist beispielsweise im Deutschen beim Präteritum der Fall, wo die Tempusendung -te fest mit der Verbform verbunden ist: ich lachte. Das Gegenteil ist etwa beim Perfekt der Fall, das analytisch gebildet wird, d. h., Hilfsverb und Hauptverb sind und bleiben voneinander getrennt: ich habe gelacht." (Hentschel/Vogel 2009: 424)

Die Relation von Hilfs- und Hauptverb wird im Rahmen der TA konsensuell - je nach Terminologie - gewöhnlich als Kopf-Kern-Relation oder als Relation von funktionalem und lexikalischem Kopf präzisiert (s. etwa Eisenberg 2005: 26f. und 2006/2: 52f. oder Primus 1997). Folgerichtig wird in der TA unter Analytisierung die Herausbildung und Grammatikalisierung von freien/ungebundenen (funktionalen) Köpfen und Kernen verstanden, die gemeinsam eine grammatische Kategorie ausdrücken.

Kein Konsens besteht dagegen darüber, ob freie Köpfe und deren Kerne Wortformen bilden. Hier hat Oliver Teuber den für das Neuhochdeutsche einleuchtenden Vorschlag gemacht, zwischen einem Flexionsparadigma, das nur die einfachen (= synthetischen) Formen eines Wortes, und einem Wortparadigma, das auch die komplexen (= analytischen) Formen enthält, zu unterscheiden (2005: 40): „Eine analytische Form ist eine syntaktisch komplexe Einheit, die als Ganzes Form eines Wortes ist. Wir können dann sagen, hat gebaut ist eine Form von bauen, ähnlich wie baute eine ist.“ (ebd.) Und weiter: „Das entscheidende Charakteristikum der analytischen Struktur ist darin zu sehen, dass das Auxiliar als Wort die Funktion hat, den paradigmatischen Wert der komplexen Form festzulegen. Anders ausgedrückt: Man benutzt ein Wort als ,Hilfswort', um in einem erweiterten Paradigma (i. e. Wortparadigma) eine neue ,Zelle' zu besetzen.“ (2005: 49) Diese (implizite oder explizite) ,Zellenbesetzungskomponente` dürfte dafür verantwortlich sein, dass in der TA Analyse und Periphrase oft - wie ich meine: vorschnell gleichgesetzt werden. Denn unter Periphrasen im engeren Sinne versteht man in 
der Sprachtypologie nach Haspelmath (2000: 656) die suppletiven Periphrasen, die entweder die Funktion haben, paradigmatische Symmetrie herzustellen (suppletive Periphrase I), oder die Funktion, ein suppletives Flexionsrealisierungsmuster für Subklassen von Lexemen zur Verfügung zu stellen (cheaper, more expensive), um ,inflectional generality“ zu erzielen (suppletive Periphrase II). Ein klassisches Beispiel für die Herstellung paradigmatischer Symmetrie sind Tempusperiphrasen des Passivs im Lateinischen:

\begin{tabular}{|l|l|l|}
\cline { 2 - 3 } \multicolumn{1}{l|}{} & Aktiv & Passiv \\
\hline Präsens & capit & capitur \\
\hline Imperfekt & capiebat & Capiebatur \\
\hline Perfekt & cepit & [captum est] \\
\hline Plusquamperfekt & ceperat & [captum erat] \\
\hline
\end{tabular}

Tab.: suppletive Periphrase I (nach Haspelmath ebd.)

Suppletive Periphrasen I sind also analytische Formen für markierte Kategorien, wenn sich ihre Herausbildung oder Grammatikalisierung durch die Herstellung paradigmatischer Symmetrie motivieren lässt. Suppletive Periphrasen II sind analytische Formen für markierte Wörter, deren synthetische Kategorienbildung blockiert ist.

Analytische Formen, die nicht suppletiv sind, sondern „some additional semantic distinction" ausdrücken und „less directly relevant to morphology" sind, nennt Haspelmath (ebd.) kategoriale Periphrasen (= Periphrasen im weiteren Sinne). Bekannte Beispiele für kategoriale Periphrasen im Deutschen sind z. B. die am-Progressivkonstruktion ((X) ist am überlegen...) oder kommen + Partizip II des Typs (X) kommt gerannt als „ein regelhaftes analytisches Mittel zum Ausdruck von räumlicher Determiniertheit" (Vogel 2005: 61f.)

Fazit: In der Diskussion zwischen NSM und TA scheint es nicht nur um die theoretische Interpretation der Daten, sondern auch um die begriffliche Hoheit über Termini zu gehen. Analyse wird im NSM als ,Noch-mehr-Synthese' uminterpretiert. Dabei wäre es überhaupt nicht notwendig gewesen, einen neuen Synthesebegriff einzuführen, um den besonderen Status der syntagmatischen Verdichtung zu betonen. Man hätte auch den Begriff der Komplexität (z. B. Fischer 2007) wählen können, der den Sachverhalt präzise getroffen hätte, ohne terminologische Verwirrung zu stiften.

3.2 Der Begriff des kombinierenden (diskontinuierenden) morphologischen Sprachtyps

Wie aus Abbildung 1 oben ersichtlich, wird der kombinierende Sprachtyp spiegelbildlich zum flektierenden dargestellt: Beim flektierenden Typus ist es der Ausdruck, der Kategorien vereint, beim kombinierenden ist es umgekehrt die 
Kategorie, die Ausdrücke zusammenführt. Diese Darstellung ist allerdings trügerisch. Denn die Ausdruck-Kategorie-Relation bezieht sich bei den ,traditionellen“ Typen ,isolierend', ,agglutinierend' und ,flektierend' auf die paradigmatische Kodierung von grammatischen Kategorien und nicht wie beim kombinierenden Typus einerseits auf die syntagmatische Kombination von grammatischen Kategorien - syntagmatische Disambiguierung, Typus d-er schön-en Katze-n -, andererseits auf die paradigmatische Kodierung von grammatischen Kategorien - herkömmliche analytische Formen (= Periphrasen im Sinne des NSM), Typus wird gebaut. Das vordergründige Problem mit dem Begriff des kombinierenden morphologischen Sprachtyps scheint demnach, dass er nicht einen Typ, sondern zwei Typen darstellt.

Reicht es, um das Problem zu lösen, den kombinierenden Typus in zwei Typen - syntagmatische Disambiguierung und Periphrase im Sinne des NSM - zu splitten, oder liegt das Problem tiefer?

Um diese Frage zu beantworten, sollen hier beide Typen - zuerst das Verfahren der syntagmatischen Disambiguierung, dann Periphrase im Sinne des NSM näher betrachtet werden (3.2.1 und 3.2.2). Anschließend wird der Frage nach dem Verhältnis von Kombinatorik und Klammerung nachzugehen sein (3.2.3). Schließlich soll mit Hilfe der Diskussionsergebnisse die zentrale Frage nach Synthese vs. Analyse beantwortet werden (3.2.4).

\subsubsection{Das Verfahren der syntagmatischen Disambiguierung}

(1) Alle Konstituenten einer Wortgruppe wie d-er schön-en Katze-n sind flektierend, sie alle entsprechen dem Schema eines flektierenden Sprachtyps nach Abbildung 1. Nach diesem Schema ist auch der Typus la-s casa-s roja-s flektierend. Der Unterschied, auf den auch das NSM abhebt, besteht in der kontinuierlichen vs. diskontinuierlichen Kategorienrealisierung (Kongruenz vs. Diskontinuität), also in den auf der Flexion basierenden unterschiedlichen syntagmatischen Relationen. M. a. W., es geht hier nicht um zwei morphologische Sprachtypen, sondern um zwei syntaktische Pole der Kategorienrealisierung, die von mehr oder weniger kontinuierlichen bis zur diskontinuierlichen Kategorienrepräsentation reichen. Daraus folgt, dass eine radikale Gegenüberstellung von Kongruenz und Kombinatorik bzw. die vom NSM suggerierte unidirektionale Entwicklung zumindest fragwürdig sind. Es stellt sich nämlich die Frage, was eigentlich die theoretisch-methodische Basis sein könnte, den Fall la-s casa-s roja-s als Paradefall der Kongruenz und den Fall d-er schön-en Katz̧e- $n$ als Paradefall der Nicht(mehr)kongruenz aufzufassen. Denn der spanische Fall ist ein radikales Beispiel für Flexionsabbau, bei dem in der Substantivgruppe nur noch der Numerus flexivisch kodiert wird (Kongruenz als Kategorienidentität). Im Vergleich zu ihm war Lateinisch morphologisch synkretischer (,ambiger'), ,kombinierender ${ }^{6}$ (Kongruenz als Kategorienüberlappung). 
Der Fall $d$-er schön-en Katze-n $n$ ist dagegen kein Fall für Flexionsabbau, sondern einer für Synkretismuszunahme (Zunahme der Kategorienüberlappung). Sein ahd. oder germ. Kongruenz-Vorgänger steht also in keinem erkennbaren theoretischmethodischen Zusammenhang mit dem als Modell benutzten spanischen Kongruenz-Typus.

Fazit: Von einem Typenwandel von Kongruenz (Ahd.) zur Kombinatorik (Nhd.) ließe sich (im syntagmatischen Sinne) tatsächlich sprechen, denn es ist nicht unplausibel anzunehmen, dass sich die nachweislich radikale quantitative Zunahme der Synkretismen im Deutschen ab einem bestimmten historischen Punkt als strukturell-qualitativer Umschwung interpretieren lässt. Allerdings wird diese (plausible) Interpretationsmöglichkeit ausgerechnet im NSM ausgeschlossen, indem Flexionsabbau mit Synkretismuszunahme bzw. Kategorienidentität mit Kategorienüberlappung verwechselt werden.

(2) Die Beschränkung der Disambiguierungsproblematik auf die Relation von eindeutigen vs. unterdeterminierten (,ambigen') Morphemen, d. h. auf bloße Kodierungsmuster, stellt eine inadäquate Reduktion des Phänomens dar. Nach dem NSM wären etwa dt. ich lieb-e und lat. ego am-o gleichermaßen kongruierend (= Kodierungsmuster ,eindeutig-eindeutig), umgekehrt wären $d$-ie Frau-O und $d$-ie Frau-en gleichermaßen kombinierend (= Kodierungsmuster ,ambig-ambig). Dabei haben, was die Art der syntagmatischen Verkettung anbelangt, eher ich lieb-e und d-ie Frau-0 bzw. ego am-o und d-ie Frau-en strukturelle Gemeinsamkeiten, die sich aber nicht als Interdependenz-, sondern als Dependenzrelationen beschreiben lassen, vgl.

(1) *(Ich) liebe $(\mathrm{X})$.

(2) *(Der) Stuhl ist bequem.

(3) (Ego) amo (X).

(4) (Die) Stühle sind bequem

(1) und (2) exemplifizieren die exzentrische, (3) und (4) die konzentrische Kodierungstechnik:: ${ }^{1}$ Die Voraussetzung der Realisierung der Verbform in (1) und der appellativischen Substantivform in (2) ist die Realisierung des Subjektspronomens bzw. des Artikels, während in (3) und (4), umgekehrte‘ Dependenzrelationen vorliegen. Dabei ist hier die Realisierung von Subjektspronomen und Artikel strukturell optional, was dazu führt, dass sich die Frage nach Kongruenz oder Kombination im strukturellen Normalfall gar nicht stellt. Im NSM spielt lediglich das Kodierungsmuster eine Rolle, die (sprachtypologisch nicht weniger relevante) Kodie-

1 Die Begrifflichkeit, die mit Makro-/Mikrovalenzrealisierung, -/+pro-drop und dependent-/headmarking verwandt ist (s. ausführlicher Ágel 2000), geht auf den Sprachtypologen Tadeusz Milewski (1967: 70ff.) zurück. Seine Unterteilung in exzentrische und konzentrische Sprachen basiert darauf, ob die grundlegenden syntaktischen Funktionen formal an Gliedern außerhalb des Prädikats oder an ins Prädikat inkorporierten pronominalen Flexiven gekennzeichnet werden. Übertragen wurden die Begriffe ,exzentrisch` und ,konzentrisch` auf die Beschreibung der Struktur der Nominalgruppe in Ágel 2006. 
rungstechnik, auf der die Kodierungsmuster aufbauen und vor deren Hintergrund sie daher überhaupt erst sinnvoll interpretiert werden können, wird ausgeklammert. Würde man auch die Kodierungstechnik mit einbeziehen, könnten hinter den kodierungsmusterbezogenen Unterschieden zwischen Deutsch und Englisch grundlegende Gemeinsamkeiten hinsichtlich der Kodierungstechnik entdeckt werden:

(5) $\quad *(\mathrm{I})$ love $(\mathrm{X})$

(6) *(The) chair is comfortable.

(7) (The) chairs are comfortable.

Nach Abbildung 1 ist Englisch isolierend, Deutsch kombinierend. Doch gerade hinsichtlich der syntagmatischen Disambiguierung scheinen diese Einstufungen nachrangig zu sein. Entscheidend ist hierfür vielmehr, was im strukturellen Normalfall realisiert werden muss und was strukturell-kodierungstechnisch optional ist. Erst auf diesem Hintergrund können die Fragen nach +/-Kongruenz und $+/$-Diskontinuität gestellt werden.

(3) Auch unabhängig von dem Problem der Kodierungstechnik stellt sich die Frage, ob es angemessen ist, in das syntagmatische Disambiguierungsverfahren gleichermaßen strukturell notwendige wie optionale Elemente mit einzubeziehen (s. d-er schön-en Katz̨e-n). Da Adjektivattribute grundsätzlich optional sind, muss das Disambiguierungspotenzial der Nominalgruppe von der Realisierung von Adjektivattributen unabhängig sein. Dort, wo das Adjektivattribut strukturell notwendig ist, hat es nicht die Funktion zu disambiguieren, sondern die exzentrische Kodierungstechnik zu ermöglichen (s. *eine Tasse Kaffees vs. eine Tasse heißen Kaffees). Nebenbei: Auch zu der im NSM zu Recht berücksichtigten Tokenfrequenz als potentiellem Synthesefaktor dürfte die Einbeziehung der Adjektivattribute kaum passen, da davon auszugehen ist, dass Nominalgruppen mit Adjektivattributen weniger frequent sind als ohne.

(4) Grundsätzlich stellt sich die Frage, ob es bei der Suche nach einer adäquaten Beschreibung des ,neuhochdeutschen Sprachtyps' sinnvoll ist, die kategoriale Disambiguierung auf die kongruierend-kombinierende Ebene der Syntagmatik zu beschränken. Wenn man das tut, kann man etwa das Englische nur negativ bestimmen: weder kongruierend noch diskontinuierend. Aber auch beim Neuhochdeutschen stellt sich die Frage, ob man auf diese Weise der Struktur der Sprache gerecht wird, vgl.

(8) Bücher sind spannend.

(9) Sie liest Bücher.

(10) Peter war von Anfang an klar, dass...

(11) Maria gefiel die Gärtnerei.

(12) Das Kind untersucht die Ärztin.

(13) Das Kind wird von der Ärztin untersucht.

(14) Die Ärztin wird vom Kind untersucht. 
(15) „So ist das Hochzeitsfest in Athen zum Politikum geworden.“ (FAZ 01.07.95: 11)

Der Kasus der konzentrischen Form Bücher bleibt in (8) und (9) strukturell offen (= ,ambig), als sog. Direktkasus kann er gar nicht disambiguiert werden, was aber unproblematisch ist, weil die jeweiligen syntaktischen Funktionen (Subjekt und direktes Objekt) qua Valenz/Konstruktion eindeutig identifiziert werden können.

Noch mehr Uniformität und noch weniger Allomorphie weist die onymische „Sparflexion“ auf (Nübling 2005: 38f.). Obwohl in Fällen wie (10) und (11) der Kasus (= Nicht-Gen.) strukturell noch offener ist als in (8) und (9) und obwohl die konzentrischen Nominalgruppen Peter und Maria - ähnlich Bücher - kongruierend-kombinierend überhaupt nicht disambiguiert (zu) werden (brauchen), kann die syntaktische Funktion (indirektes Objekt) auch hier eindeutig identifiziert werden.

In (12) hilft Position, und sollte diese zu konfligierenden Interpretationen führen können, bleibt immer noch die Möglichkeit auf (13) oder (14) auszuweichen. ${ }^{2}$

Diese Faktoren scheinen mir bei der Beurteilung des kombinatorischen Potenzials einer Sprache nicht weniger wichtig zu sein als die Kombinatorik qua Diskontinuität (zu Deutsch/Englisch s. Fischer 2007; zur strukturellen Offenheit s. Ágel 2009).

\subsubsection{Periphrasen im Sinne des NSM}

Folgendes Zitat macht deutlich, mit welcher Begründung Periphrasen im Sinne des NSM (= analytische Verbformen nach der TA) zum kombinierenden Sprachtyp gerechnet werden: „Die Information ,Passiv“ ergibt sich erst durch die Kombination aus einer Form von werden + dem Partizip II. Stünde hier ein Infinitiv, so ergäbe sich kombinatorisch die Futurlesart: sie wird sehen." (Nübling 2010: 11)

Die Überlegung ist also, dass auch hier ein Disambiguierungsfall vorliegt: Das Hilfsverb ist ambig, die Form des Vollverbs auch, kombiniert sind sie jedoch disambiguiert. Doch die Analogie mit Gruppen wie sie komm-t oder d-er schön-en Katze-n trügt. Während beim Typus der syntagmatischen Disambiguierung die (strukturell notwendigen) Elemente der jeweiligen Gruppen über kongruierbare/kombinierbare Einheitenkategorisierungen wie ,Person' und ,Numerus' bzw. ,Numerus` und ,Kasus`verfügen, verfügen die Elemente von Gruppen wie wird gesehen oder wird sehen nicht über kombinierbare Einheitenkategorisierungen wie ,Genus verbi‘ bzw. ,Tempus‘. Hier handelt es sich also nicht um syntagmatische Disambiguierung vorhandener Kategorien, sondern um genuine (paradigmatische) Kategorienbildung qua Statusrektion. Folglich kann Periphrase im Sinne des NSM

2 Damit soll natürlich nicht behauptet werden, dass Konflikte immer strukturell zu lösen sind. Um Beleg (15) interpretieren zu können, muss man den FAZ-Aufsatz gelesen haben, aus dem hervorgeht, dass der griechische Kronprinz Paul eine amerikanische Studentin in London geheiratet hat. 
weder mit syntagmatischer Verdichtung noch mit Informationsbündelung etwas zu tun haben. Da im NSM „Synthese als Form der Informationsbündelung“ (Szczepaniak 2010: 134) aufgefasst wird, scheidet die Periphrase im Sinne des NSM als synthetisches Verfahren aus. Diese Formen bleiben, was sie waren: analytische Verbformen.

\subsubsection{Kombinatorik und Klammerung}

Eine Möglichkeit, den kombinatorischen Sprachtyp auf eine einheitliche begriffliche Grundlage zu stellen, wäre vielleicht das als typologisches Merkmal des Deutschen geltende Klammerungsprinzip. Diese Möglichkeit wird im NSM auch angedeutet: Unter Umständen können die beiden ambigen Ausdrücke sogar beträchtliche Klammerkonstruktionen bilden, d.h. ausgedehnte Mittelfelder umfassen, etwa wenn zwischen Artikel und Substantiv längere Attribute treten: der [kleinen und noch nicht stubenreinen] Hunde/Katze/ Katzen. (Nübling 2010: 10)

Zu Recht schränkt hier Nübling die Parallelisierung von Kombinatorik und Klammerung ein, denn:

1. Die Bildung von Klammerkonstruktionen gilt auch für im Sinne des NSM kongruente Ausdrücke: des [kleinen und noch nicht stubenreinen] Hundes.

2. Umgekehrt gilt sie aber weder für kongruente noch für diskontinuierende (!) Subjektspronomen-Verb Verbindungen:

(16) *Du immer spät nach Hause kommst.

(17) *Sie immer spät nach Hause kommen.

3. Sie gilt jedoch für Periphrasen im Sinne des NSM (= analytische Verbformen):

(18) Du bist immer spät nach Hause gekommen.

4. Sie gilt aber auch für die Adverb- und die sog. Nebensatzklammer, die ganz oder teils auf nichtflektierbaren Elementen basieren (zur Fragwürdigkeit des Begriffs der Nebensatzklammer s. Ágel 2000a):

(19) Wo hast du die Pistole her?

(20) ...dass sie immer spät nach Hause kommt.

5. Durch die Parallelisierung von Kombinatorik und Klammerung ließe sich nicht erklären, warum es im Englischen nur eine rudimentäre Klammerbildung gibt (Bespiele von Klaus Fischer):

(21) Where have you got your pistol from?

(22) Can you put my friend up?

6. Umgekehrt ließe sich nicht erklären, warum sich in einer agglutinierenden Sprache wie dem Ungarischen trotz unflektierbarem Artikel und Adjektivatt- 
ribut genauso ausgedehnte Nominalklammern bilden lassen wie im Deutschen:

(23)
A
[kicsi és még nem szobatiszta]
kutya
$\mathrm{ART}_{\mathrm{DEF}} \quad$ klein und noch nicht stubenrein
Hund

3.2.4 Zwischenfazit: Was bleibt von der syntagmatischen Synthetisierung übrig?

Periphrase im Sinne des NSM ist eine statusrektionale Technik der paradigmatischen Kategorienbildung. Als paradigmatisches Verfahren kann sie weder syntagmatisch verdichtend noch informationsbündelnd sein.

Das Verfahren der syntagmatischen Disambiguierung stellt in Kombination mit anderen Disambiguierungsverfahren eine flexionsbasierte Technik syntagmatischer Kategorienrealisierung dar, gdw. die Kodierungstechnik exzentrisch ist und wenn die Elemente des Syntagmas strukturell notwendig sind. Da die Kodierungstechnik keinesfalls immer exzentrisch ist (ausführlich s. Ágel 2006), da es optionale und $\mathrm{u}$. U. wenig frequente syntagmatische Elemente gibt, da es - je nach modellinterner Interpretation von Kongruenz/Diskontinuität (s. Abschnitt 2) bei den einzelnen Datengruppen der syntagmatischen Disambiguierung auch ein mehr oder weniger hoher Anteil von kongruierenden Konstruktionen vorhanden ist und da die kongruierenden Konstruktionen nicht weniger verdichtend und informationsbündelnd sind als ihre diskontinuierenden Paradigmennachbarn, kann dem Verfahren der syntagmatischen Disambiguierung qua Kombinatorik keine strukturell-typologisch exklusive syntagmatische Verdichtung und Informationsbündelung zugesprochen werden. Diskontinuität ist gewiß ein wichtiges Merkmal des Neuhochdeutschen und ein wichtiges Verfahren der Informationsbündelung, aber im Sinne des Gesagten wäre es ein theoretischer Kurzschluss, hier eine strukturell-typologisch genuine Synthetisierungstendenz zu sehen. Genauso wenig lässt sich eine direkte Verbindung zwischen (Nicht-)Redundanz und Synthesegrad feststellen:

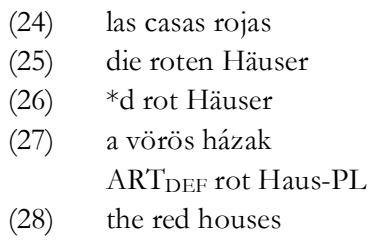

Hinsichtlich der von Nübling herangezogenen Struktur (s. Abschnitt 2) ist Deutsch genauso redundant wie Spanisch. Die Eliminierung der Redundanz im Deutschen (s. (26)) ,ergäbe' ungarische oder englische Strukturen, die maximal nichtredundant sind. Diskontinuität fördert zwar die Informationsbündelung, baut aber nicht notwendigerweise Redundanz ab. Umgekehrt liegt bei den agglutinierenden 
bzw. isolierenden Pendants, die man gewiß nicht als gleich synthetisch ansehen möchte, keine Redundanz vor.

4. Morphologische Sprachtypen und die Modellierung des Sprachwandels

Kombination/Diskontinuität ist - ähnlich der Klammerung - kein morphologischer Sprachtyp und auch kein strukturell-typologisches Synthesemerkmal, sondern eine im Deutschen besonders ausgeprägte Technik der relativ eindeutigen Kategorienrealisierung, die den flektierenden morphologischen Sprachtyp voraussetzt. Bemerkenswert an Abbildung 1 (s. Abschnitt 2) ist, dass Nübling nicht, wie in der Sprachtypologie üblich, den flektierenden Typus, sondern den kombinierenden fusionierend nennt, was möglicherweise auch terminologisch unterstreichen soll, dass der kombinierende Typus fusionierender ist als der in der Sprachtypologie fusionierend genannte.

Seit Sapir (1921) rechnet man bei morphologischen Typologien mit zwei Kriterien, deren Anwendung zu nichtparallelen Klassifikationen der ,klassischen“ Typen führt: Synthese und Fusion (ausführlicher s. etwa Croft 1990: 39ff.).

Mit Synthese wird die morphologische Komplexität von Wörtern erfasst. Nach diesem Kriterium sind alle morphologischen Typen außer, isolierend' (mehr oder weniger) synthetisch.

Unter (morphologischer) Fusion versteht man die +/- Portmanteau-Realisierung von Morphemen im Wort. Dieses Kriterium lässt sich am besten für die Unterscheidung von agglutinierenden und flektierenden (eben: fusionierenden) bzw. - mit Einschränkungen - introflexiven Sprachen anwenden. Bei isolierenden (und polysynthetischen) Sprachen würde die Anwendung des Kriteriums zu einer Binnengliederung des jeweiligen Typs führen.

Die analytischen Formen im Sinne der TA lassen sich mit dem Kriterium der Fusion nicht erfassen. Sie stellen weder +- noch - Portmanteau-Realisierungen dar. Hinsichtlich des Kriteriums der Synthese stehen sie zwischen den traditionellen synthetischen Strukturen mit morphologisch komplexen Wörtern und den isolierenden Strukturen mit morphologisch einfachen: Analytische Formen bilden eine - je nach Theorie - morphologisch/syntaktisch komplexe Gestalt (Wort, speech unit, dissoziierten Nukleus, Phrase, Konstruktion) mit analogen grammatischen Funktionen, wie sie in synthetischen Strukturen mit morphologisch komplexen und in isolierenden Strukturen mit morphologisch einfachen Wörtern ausgedrückt werden. Analytische Strukturen sprengen also den traditionellen Wortrahmen, nutzen jedoch in ihrem (wie auch immer definierten), neuen Rahmen' sowohl Einfachheit als auch Komplexität.

Will man die traditionelle morphologische Typologie nicht radikal reformieren (was in der Sprachtypologie permanent geschieht, s. Bickel/Nichols 2005 und 2005a), könnte man einerseits Synthese/Analyse als Leitkriterium nehmen, ande- 
rerseits auch (morphologische) Suppletivität (= suppletive Periphrastik) als morphologischen Sprachtyp mit Sonderstatus mit einbeziehen. Suppletivität ist ein morphologischer Sprachtyp, weil suppletiv-periphrastische Strukturen in paradigmatischer Relation zu nichtsuppletiven (= agglutinierenden, flektierenden und isolierenden) stehen, die man morphologischen Sprachtypen zuordnet. Der Sonderstatus besteht darin, dass suppletive Strukturen syntagmatisch organisiert sind.

Was genau ist nun unter dem suppletiven morphologischen Typ zu subsumieren?

Sichere Kandidaten sind die suppletiven Periphrasen (I und II). Sicher ist umgekehrt, dass sonstige analytische Strukturen, d. h. kategoriale Periphrasen (s. (X) kommt gerannt, (X) ist am überlegen), nicht suppletiv sind, sondern nur mögliche Vorstufen von suppletiven Periphrasen darstellen. Zwischen den suppletiven und kategorialen Periphrasen scheint es allerdings noch ein Übergangsgebiet zu geben, das Haspelmaths Theorie nicht abdeckt: Analytische Formen, die keine paradigmatischen Lückenfüller sind, aber auch nicht einfach „some additional semantic distinction“ ausdrücken und schon gar nicht „less directly relevant to morphology" sind (Haspelmaths Definition der kategorialen Periphrasen, s. 3.1). Man denke etwa an die periphrastischen Passive und Tempora im Deutschen. Auch diese Formen sind morphologisch suppletiv, aber sie haben (in der Regel) nicht die Funktion, paradigmatische Symmetrie herzustellen, sondern Paradigmen Wortparadigmen im Teuber'schen Sinne - zu bilden.

Vorsichtshalber sollte ausdrücklich betont werden, dass die Redeweise von Sprachtypen im doppelten Sinne eine sehr abstrakte ist. Einerseits sind die Grenzen zwischen den einzelnen Typen, besonders zwischen ,agglutinierend' und ,flektierend', nicht auf einfache Dichotomien zu reduzieren (s. Plank 1999). Andererseits sind es bekanntlich nicht ganze Sprachen/Sprachstufen, sondern nur bestimmte Konstruktionen, die ,agglutinierend', ,flektierend', ,suppletiv' usw. sind (Comrie 1996). Redet man von Sprachtypen, meint man also, dass ein bestimmter (tendenziell agglutinierender, flektierender, suppletiver usw.) Konstruktionstyp in der jeweiligen Sprache/Sprachstufe auffallend präsent ist. „Die Systemtypologie soll deshalb durch eine Typologie der parole ergänzt werden." (Fischer 2007: 401)

Um dem potentiellen Sprachwandel-Kreislauf, den realen strukturellen Verwandtschaften zwischen den germanischen Sprachen/Sprachstufen und den komplizierten Analyse/Synthese-Relationen einigermaßen gerecht zu werden, wird in Abbildung 2 nicht eine lineare Anordnung der Sprachtypen wie in Abbildung 1, sondern eine kreisförmige gewählt. Dabei bleiben auch hier wie in Abbildung 1 der polysynthetische und der introflexive Typ unberücksichtigt: 


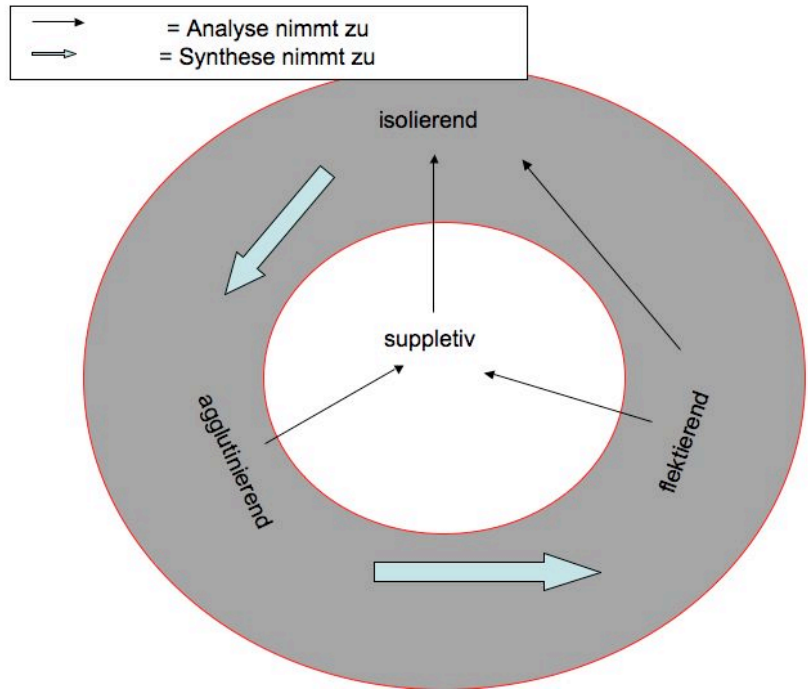

Abb. 2: morphologische Sprachtypen

Die Abbildung versucht insbesondere folgende Aspekte zu visualisieren:

(1) Dadurch, dass der suppletive Typus aus dem ,Kreisfluss` herausgenommen wird, soll sein Sonderstatus angedeutet werden. Dass er optisch in der Mitte steht, ist zufälliges Nebenprodukt der Darstellung und bedeutet nicht, dass der Typus zentral wäre.

(2) Durch die räumliche Trennung des suppletiven Typus von den nichtsuppletiven Typen, scheint es möglich, Analytisierungs-/Synthetisierungsrichtungen und Sprach-/Strukturwandelrichtungen zumindest in Bezug auf das Germanische mit denselben Pfeilen anzuzeigen. Wäre der generelle Sprach-/Strukturwandel bezüglich Analytisierung/Synthetisierung grundsätzlich bidirektional, müsste ,ist weniger analytisch als' eine Umkehrrelation zu ,ist weniger synthetisch als' sein und zu jedem einfachen Pfeil in Abbildung 2 ein umgekehrter doppelter Pfeil verlaufen (und vice versa). Mit Abbildung 2 wird allerdings nicht der Anspruch verbunden, diese Frage angesichts der ca. 6000 Sprachen zu klären.

(3) Die räumliche Trennung macht es auch möglich, darauf hinzuweisen, dass Suppletivität sowohl auf agglutinierenden als auch auf flektierenden Strukturen funktioniert, aber

(4) auf Isolierungsbasis (logisch) wohl ausgeschlossen ist. Dies bedeutet allerdings nicht, dass primär isolierende Sprachen keine suppletiven Strukturen haben können, sondern nur, dass sich diese auf der Basis von früheren flektierenden oder agglutinierenden und nicht auf der Basis von isolierenden Strukturen herausbilden. 
Nach Abbildung 1 ist das Germanische dem agglutinierenden Typus zuzuordnen. Von hier aus führt der Weg zu den älteren Sprachstufen des Deutschen und Englischen, die primär flektierend sind. Hier trennen sich partiell die Wege von Englisch und Deutsch gemäß Abbildung 2 wie folgt:

Englisch durchläuft eine ,doppelte` Analytisierung: (a) Richtung ,isolierend und (b) Richtung , suppletiv'. Modernes Englisch ist daher tendenziell suppletivisolierend. Deutsch dagegen ist weniger radikal, die Analytisierung verläuft nur in einer Richtung. Neuhochdeutsch ist primär flektierend-suppletiv. ${ }^{3}$

\section{Literatur}

Ágel, Vilmos (2000): Valenztheorie. Tübingen: Narr (Narr Studienbücher).

--- (2000a): Syntax des Neuhochdeutschen bis zur Mitte des 20. Jahrhunderts. In: Besch, Werner/Betten, Anne/Reichmann, Oskar/Sonderegger, Stefan (Hg.): Sprachgeschichte. Ein Handbuch zur Geschichte der deutschen Sprache und ihrer Erforschung. 2. Aufl. Bd. 2. Berlin/New York: Walter de Gruyter (HSK 2.2), 1855-1903.

--- (2006): (Nicht)Flexion des Substantiv(s). Neue Überlegungen zum finiten Substantiv. In: ZGL 34, 286-327.

--- (2009) Strukturelle Offenheit mit Verstehenspräferenzen. Plädoyer für eine Neuorientierung in der Erforschung globaler Ambiguitäten. In: Linke, Angelika/Feilke, Helmuth (Hg.): Oberfläche und Performanz. Untersuchungen zur Sprache als dynamischer Gestalt. Tübingen: Niemeyer (RGL 283), 137-159.

Bickel, Balthasar/Nichols, Johanna (2005): Fusion of Selected Inflectional Formatives. In: Haspelmath, Martin/Dryer, Matthew S./Gil, David/Comrie, Bernard (Hg.): The World Atlas of Language Structures. Oxford: Oxford University Press.

--- (2005a): Exponence of Selected Inflectional Formatives. In: Haspelmath, Martin/Dryer, Matthew S./Gil, David/Comrie, Bernard (Hg.): The World Atlas of Language Structures. Oxford: Oxford University Press.

Comrie, Bernard (1996): Sprache und Sprachen. Universalien und Typologie. In: Lang, Ewald/Zifonun, Gisela (Hg.): Deutsch - typologisch. Jahrbuch 1995 des Instituts für deutsche Sprache. Berlin/New York: Walter de Gruyter, 16-30.

Croft, William (1990): Typology and Universals. Cambridge: Cambridge University Press. (Cambridge Textbooks in Linguistics).

Eisenberg, Peter (2005): Das Verb als Wortkategorie des Deutschen. Zum Verhältnis von synthetischen und analytischen Formen. In: Knobloch, Clemens/Schaeder, Burkhard (Hg.): Wortarten und Grammatikalisierung. Perspektiven in System und Erwerb. Berlin/New York: Walter de Gruyter, 21-41.

--- (2006/2): Grundriss der deutschen Grammatik. Bd. 2: Der Satz. 3. Aufl. Stuttgart/Weimar: Metzler.

Fischer, Klaus (2007): Komplexität und semantische Transparenz im Deutschen und Englischen. In: Sprachwissenschaft 32, 355-405.

3 Für kritische Lektüre, zahlreiche Anregungen und wertvolle Kommentare danke ich Gisela Zifonun, Klaus Fischer und Andreas Nolda. 
Haspelmath, Martin (2000): Periphrasis. In: Booij, Geert/Lehmann, Christian/Mugdan, Joachim (Hg.): Morphologie. Ein internationales Handbuch zur Flexion und Wortbildung. Halbbd. 1. Berlin/New York: Walter de Gruyter (HSK 17.1), 654-664.

Hentschel, Elke/Vogel, Petra M. (Hg.) (2009): Deutsche Morphologie. Berlin/New York: Walter de Gruyter (de Gruyter Lexikon).

Milewski, Tadeusz (1967): La structure de la phrase dans les langues indigènes de l'Amérique du Nord. In: Ders. Études typologiques sur les langues indigènes de l'Amérique. Kraków: Polska Akademia Nauk ( Prace komisji orientalistycznej 7), 70-101. [Orig. 1950]

Nübling, Damaris (2005): Zwischen Syntagmatik und Paradigmatik: Grammatische Eigennamenmarker und ihre Typologie. In: ZGL 33, 25-56.

--- (2010): Lässt sich ein Syntheseindex erstellen? Zur Problematisierung und Präzisierung eines (allzu) geläufigen Begriffs. In: Bittner, Dagmar/Gaeta, Livio (Hg.): Kodierungstechniken im Wandel. Das Zusammenspiel von Analytik und Synthese im Gegenwartsdeutschen. Berlin/New York: Walter de Gruyter, 1-22.

Plank, Frans (1999): Split morphology: How agglutination and flexion mix. In: Linguistic Typology 3, 279-340.

Primus, Beatrice (1997): Der Wortgruppenaufbau in der Geschichte des Deutschen: Zur Präzisierung von synthetisch vs. analytisch. In: Sprachwissenschaft 22, 133-159.

Ronneberger-Sibold, Elke (1980): Sprachverwendung - Sprachsystem. Ökonomie und Wandel. Tübingen: Niemeyer.

Sapir, Edward (1921): Language. An Introduction to the Study of Speech. New York: Harcourt, Brace \& World, Inc.

Schwegler, Armin (1990): Analyticity and Syntheticity. A dDachronic Perspective with Special Reference to Romance Languages. Berlin/New York: Mouton de Gruyter.

Szczepaniak, Renata (2010): Wird die deutsche Nominalphrase wirklich analytischer? Zur Herausbildung von Diskontinuität als synthetische Verdichtung. In: Bittner, Dagmar/Gaeta, Livio (Hg.): Kodierungstechniken im Wandel. Das Zusammenspiel von Analytik und Synthese im Gegenwartsdeutschen. Berlin/New York: Walter de Gruyter, 123-136.

Teuber, Oliver (2005): Analytische Verbformen im Deutschen. Syntax - Semantik Grammatikalisierung. Hildesheim/Zürich/New York: Olms (GL 18).

Vogel, Petra M. (2005): Neue Überlegungen zu den Fügungen des Typs sie kamen gelaufen. In: ZGL 33, 57-77.

Werner, Otmar (1979): Kongruenz wird zu Diskontinuität im Deutschen. In: Brogyanyi, Bela (Hg.): Studies in Diachronic, Synchronic, and Typological Linguistics. Festschrift für Oswald Szemerényi, Bd. 2. Amsterdam/Philadelphia: Benjamins, 959-988.

\author{
Adresse des Verfassers: \\ Prof. Dr. Vilmos Agel, Fachbereich 02 - Germanistik, Universität Kassel, Kurt-Wolters-Str. 5, \\ D-34125 Kassel. \\ E-Mail:agel@uni-kassel.de
}

